\title{
Appropriate research in IT in emerging countries
}

\author{
Paul Nicholson \\ Deakin University \\ Burwood \\ VIC Australia 3125 \\ E-mail:Pauln@deakin.edu.au
}

\section{INTRODUCTION}

Much of the research about IT in education is essentially Western, reflecting the Western research paradigm, models of education and culture. It is essential that the embedded influences that permeate the research in undefined and intangible ways are recognised as essentially cultural artifacts.

Within the research literature itself, there is no one preferred methodology that suits all circumstances. Instead a number of competing paradigms are often represented as being 'better' or 'more reliable' or even 'more valid' than others (e.g., Lynch \& Tarson, 1984). The debate about methodologies is much more significant than the debate about specific models and methods of quantitative research, and is very difficult to engage.

The purpose of this workshop session is not to directly engage in the debate, other than to acknowledge it. You will be presented with two 'alternative' models of research that appear to have much to offer educators in many countries. Together they can facilitate effective research in implementation and in planning for IT in education (along with many other issues).

The workshop will briefly discuss action research, and engage with its essential methodological components, before moving on to examining the use of scenariobased research as a means of planning and positioning educational computing in a changing cultural, social and political milieu. It is a kind of 'action research for the future'. 
Action research is research that is embedded in the experience, context and (frequently) workplace of the researcher. Its focus is on the 'practitioner as researcher' with the outcomes being realistic understandings and solutions to practitioner-level problems. It provides iterative solutions to real problems through an ongoing cycle of research, reflection and then application of strategies derived from the research. Its major strength is the localisation of the research with the practitioner and the consequent development of increased understandings.

Action research is good for solving problems that require strong local knowledge, give flexible and adaptable outcomes, allow for localised solutions, and involve community values and attitudes to be incorporated as valid parameters.

Often action research is not valued because it is such a simple approach to solving many educational problems that it does not require the theoretical constructs that define and constrain most other models of research.

Scenario-based research is action research for the future. It is 'future-proofing' education and business. It is a rapid response plan, an informed, researched action plan to meet future challenges in policy, economic or political factors.

Most of us must make a decision now! Anything that can help make a decision in the confusion of uncertainty will be valuable. Scenario planning is one such tool.

The primary driving forces of scenario-based research are:

- social dynamics - quantitative, demographic issues;

- economic issues - macroeconomic trends and forces shaping the economy as a whole;

- political issues - electoral, regulatory and litigative;

- technological issues - direct, enabling and indirect.

After identifying the predetermined elements of the driving forces, a number of uncertainties remain. Are they critical uncertainties - an uncertainty that is key to our focal issue?

Determine the set of scenarios that focus on our uncertainty:

- Realize that there is more than one possible future.

- Understand that it is important to identify the most likely possibilities.

- Accept that the 'solution' that applies across the widest range of possible futures is probably the best and most robust.

Now we will work through the process of scenario planning. Realize that there will only be enough time to develop one scenario. The workshop is about understanding the process! 


\title{
WORKSHOP TIMETABLE
}

\author{
Phase 1: Where am I now? \\ 60 minutes
Review of current situation
Small group presentation
Writing task \\ Identification of assumptions
}

Phase 2: What can change?

60 minutes

Identification of issues

Articulating and prioritising the impact

Small group presentation

Writing task

Phase 3: Planning for change

60 minutes
Short and long term reactions
Small group presentation
Writing task

Phase 1: Where am I now?

(60 minutes)

In this phase it is essential to identify 'where you are' now.

This includes identifying the assumptions that are embedded in your current situation and thinking, and a listing of all of the factors that have led to the present.

- Briefly describe your current situation:

- In the following box, make a list of the KEY assumptions that you believe underpin 'where you are now' in regard to the situation you want to consider. 
- In the following box, list the KEY factors that have led to the present situation.

- List the THREE MOST satisfactory features of the present situation.

1.

2.

3.

- List the THREE LEAST satisfactory features of the present situation.

4.

5.

6.

- Now you can give a 5 minute presentation on your work to your group. This will help you to ensure that you have covered the key issues. At the end of the group session, you should add/revise your items above based on feedback from the group. You should then have a clear idea of where you are starting from, or at least as seen by others.

Phase 2: What can change?

(60 minutes)

The whole point about scenarios is that they confront the future. Therefore there are no certainties.

In this part of the process, it is important for you to identify issues and trends that could subtly or significantly alter the way in which you now go about your business. Some of this will come from your professional understandings of the area you are working in; other ideas will come from hunches and gut feelings.

This is a key part of scenario planning - getting your expert, intuitive ideas on paper where they can be reacted to.

- List at least 6 things that, in your opinion, could alter the present situation. 
Phase 3: Planning for change?

(60 minutes)

For each of the three issues that you listed in the table, suggest at least two appropriate ways in which you could react to them, if they became reality.

\section{Issue 1}

response 1

response 2

Issue 2

response 1

response 2

\section{Issue 3}

response 1

response 2

- Present a 5 minute summary of your proposed responses to your group. Use the feedback to refine them to your satisfaction.

- Now, take the issue that you consider to be the most significant and develop your response to it in two ways:

1. As a short term response ( $1-3$ months) so that you address its immediate concerns.

2. As a long term response (1-3 years) where you:

- show how you expect it to develop over time,

- describe how you anticipate it might change, 
- Prioritise these in order of 'most likely' to 'unlikely'.

1
2
3
4
5
6
7
8

Take the three top items and explain them to your group. To do this well, you will have to clearly identify the potential issue and explain its likely impact to the other members. The group will give you advice about the issue, and what might need to be explained or explored further. At this stage, you should be able to clearly identify exactly what the issue is and what its origins are. To make sure that you have thought this through, complete the following self-check table.

\begin{tabular}{|c||c|c|c|}
\hline Issue & Details & Comes from & Reason for its existence \\
\hline \hline 1 & & & \\
& & & \\
& & & \\
& & & \\
& & & \\
& & & \\
& & & \\
& & & \\
& & & \\
& & & \\
\end{tabular}


- the KINDS of institutional, social or economic resources that might be needed,

- social, cultural, economic and political considerations.

\section{SHORT TERM STRATEGY:}

\section{LONG TERM STRATEGY:}

At the end of the session, you will have the opportunity to present your work in a 10 minute presentation to the whole group involved in the workshop.

\section{5}

\section{REFERENCES}

Lynch, P. and Tarson, M. (1984) Research on Educational Planning: An International Perspective, in (ed. E. Gordon) Review of Research in Education, 11, American Educational Research Association, Washington DC. 